\title{
Review
}

\section{A Review of the Role of Anticholinergic Activity in Lewy Body Disease and Delirium}

\author{
Yuka Kitajima $^{a}$ Koji Hori ${ }^{f}$ Kimiko Konishi ${ }^{b, f}$ Masayuki Tanic Hiroi Tomioka ${ }^{f}$ \\ Norihisa Akashi ${ }^{d}$ Misa Hosoi ${ }^{f}$ Atsuko Inamoto ${ }^{f}$ Sayaka Hasegawa ${ }^{f}$ \\ Nodoka Kikuchi $^{f}$ Akari Takahashif $^{f}$ Mitsugu Hachisu ${ }^{e}$ \\ ${ }^{a}$ Department of Anesthesiology, School of Medicine, Juntendo University, ${ }^{b}$ Tokyo Metropolitan Tobu Medical

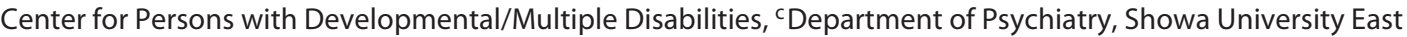 \\ Hospital, dDepartment of Psychiatry, Showa University Karasuyama Hospital, and e Department of Pharmaceutical \\ Therapeutics, Division of Clinical Pharmacy, School of Pharmacy, Showa University, Tokyo, and f Department of \\ Psychiatry, Showa University Northern Yokohama Hospital, Yokohama, Japan
}

\section{Key Words}

Alzheimer's disease $\cdot$ Delirium $\cdot$ Lewy body disease $\cdot$ Serum anticholinergic activity · Acetylcholine · Cholinesterase inhibitor - Mental stress

\footnotetext{
Abstract

We have previously proposed a hypothesis in which we argue that anticholinergic activity (AA) appears endogenously in Alzheimer's disease (AD). Acetylcholine (ACh) controls both cognitive function and inflammation. Consequently, when the downregulation of ACh reaches critical levels, the inflammatory system is upregulated and proinflammatory cytokines with AA appear. However, factors other than downregulation of ACh can produce AA; even if ACh downregulation does not reach critical levels, AA can still appear if one of these other AA-producing factors is added. These factors can include neurocognitive disorders other than $A D$, such as delirium and Lewy body disease (LBD). In delirium, ACh downregulation fails to reach critical levels, but AA appears due to the use of medicines, physical illnesses or mental stress (termed ' $A A$ inserts'). In $L B D$, we speculate that $A A$
}

appears endogenously, even in the absence of severe cognitive dysfunction, for 2 reasons. One reason is that patterns of $A C h$ deterioration are different in LBD from those in $A D$, with synergistic actions between amyloid and a-synuclein thought to cause additional or severe symptoms that accelerate the disease course. The second reason is that AA occurs through disinhibition by reduced cortisol levels that result from severe autonomic parasympathetic dysfunction in LBD.

(c) 2015 S. Karger AG, Basel

\section{Introduction}

We have previously argued that endogenous anticholinergic activity (AA) occurs in Alzheimer's disease (AD) [1-3]. In the anticholinergic hypothesis, we emphasized that downregulation of acetylcholine (ACh) could be implicated in $\mathrm{AD}$ [4], and that $\mathrm{ACh}$ also regulates the inflammatory system, making downregulation of the cholinergic system critical to the pathology of $\mathrm{AD}$. We also discussed the importance of N-methyl-D-aspartate receptor expression being upregulated, leading to activation of

\section{KARGER 125}

(c) 2015 S. Karger AG, Base

$1660-2854 / 15 / 0153-0162 \$ 39.50 / 0$

E-Mail karger@karger.com

www.karger.com/ndd
Koji Hori, MD, PhD

Department of Psychiatry, Showa University Northern Yokohama Hospital 35-1 Chigasakichuo, Tsuzukiku

Yokohama, Kanagawa 224-8503 (Japan)

E-Mail kojihori@med.showa-u.ac.jp 
Fig. 1. We speculate that ACh degradation not only causes cognitive dysfunction and behavioral and psychological symptoms of dementia (BPSD), but also induces inflammation in the central and peripheral nervous systems, which then induces AA through cytokine pathways. Moreover, AA causes an increase in amyloid and further decreases ACh. This is referred to as the 'endogenous AA cascade'. NMDA $=\mathrm{N}$ methyl-D-aspartate; SAA = serum anticholinergic activity. This figure is reproduced from an article by Hori et al. [1]. The procedure was permitted by the Japanese Society of Neuropsychopharmacology (Tokyo, Japan).

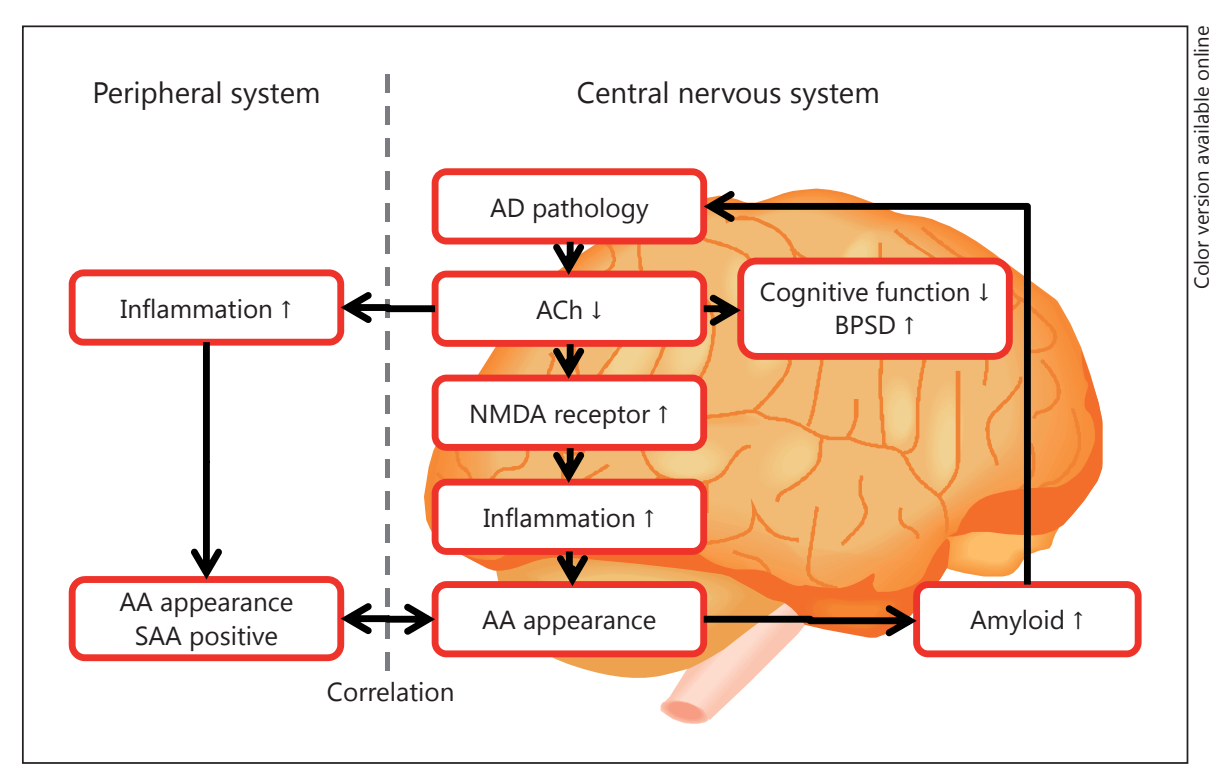

the inflammatory system. The subsequent inflammationinduced release of cytokines by activated microglia produces AA that accelerates the pathology of $\mathrm{AD}[1-3,5]$ (fig. 1; courtesy of Hori et al. [1]). It is possible that AA covers the gap between cholinergic downregulation or inflammation and the pathological changes in AD. Moreover, we also commented that serum anticholinergic activity (SAA) [6] was a useful peripheral marker of rapidly progressive $\mathrm{AD}[1,2]$. Although we have also experienced patients with mild $\mathrm{AD}$ that have had positive SAA, their cognitive decline was not sufficiently critical to elicit AA endogenously. In these instances, we speculated that SAA positivity was induced by the addition of mental stress to preexisting ACh downregulation [7]. We consider mental stress, and other factors capable of inducing SAA, to be 'AA inserts'. In this article, we describe the possibility that the presence of AA causes neurocognitive disorders such as delirium and hallucination in Lewy body disease (LBD), based on the dual actions of ACh downregulation coupled with the effects of another AA insert.

\section{Dual Inserts Can Induce AA in Other Neurocognitive Disorders}

The notion that dual inserts can induce AA in other neurocognitive disorders can be evidenced by a case report. A 74-year-old woman presented to our memory clinic complaining of memory disturbances for 2 years. She had moved to a new residence 3 months before visit- ing our clinic, which had caused excessive stress. Since the move, she had been troubled by visual hallucinations, apathy, irritability and blurred vision. On initial examination, her Mini-Mental State Examination [8] score was 26, and her SAA marker concentration was $4.28 \mathrm{nM}$. We diagnosed mild cognitive impairment [9] with AD using a scale developed by the National Institute of Neurological and Communicative Disorders and the Stroke and Alzheimer's Disease and Related Disorders Association working group [10]. The diagnosis was made on the basis that her cognitive function was relatively preserved, and neither she nor her husband had noted the disturbances in the activity of her daily life. We therefore prescribed donepezil ( $5 \mathrm{mg}$ daily), which led to a prompt resolution of her visual hallucinations, blurred vision and apathy. By 1 year of treatment, her Mini-Mental State Examination score had decreased to 24, and her SAA had become undetectable $(<1.95 \mathrm{nM})$.

In this case report, we discussed that the appearance of AA might be caused by either of 2 patterns of AD pathology [3]. It seems that exogenous factors (AA inserts), such as medication, illness and mental stress, with minimal ACh downregulation can lead to the development of AA as occurred in this patient. From this case presentation, we reported that 2 patterns might be associated with the production of $\mathrm{AA}$ in $\mathrm{AD}$ [3]. In one pattern, endogenous $\mathrm{AA}$ triggers a rapid cognitive decline from moderate $\mathrm{AD}$ when the level of ACh reaches a critical level. In the other pattern, cognitive decline begins without ACh downregulation needing to reach a critical level; instead, AA follows 
minimal ACh downregulation due to medication, illness or mental stress [3]. Delirium can therefore result, even if ACh downregulation does not reach the critical level, because AA appears as the result of the AA insert. We speculate that this occurs in neurocognitive disorders such as delirium and LBD.

\section{Delirium and AA}

We have previously evaluated the relationship between postoperative delirium and SAA. Although delirium is considered an important issue among elderly patients in emergency departments, surgical and oncology wards, intensive care units and nursing homes, the mechanism underlying delirium is poorly understood [11]. Described as an acute and fluctuating disturbance of consciousness, it has been reported to contribute to an increase in discharge mortality and to prolonged hospital stays [12]. Among the mechanisms postulated to cause delirium, such as neurotransmitter changes, inflammation, physiological stress, metabolic derangement, electrolyte disorders and genetic factors [13], cholinergic failure is often cited as the most influential [14]. Given that it is difficult to evaluate cholinergic neuronal activity in the brain directly, SAA levels have been used as a surrogate marker of central AA [15].

We previously demonstrated that there was a relationship between perioperative SAA and the occurrence of postoperative delirium [16]. Using the radioreceptor technique described by Tune and Coyle [6], the SAA level of the blood samples was measured and expressed as atropine equivalents. We then categorized a level $\geq 1.95 \mathrm{nM}$ as SAA,$+<1.95 \mathrm{nM}$ as SAA \pm and an undetectable level as SAA- [2]. We included the SAA \pm class because a low SAA can induce AA in the central nervous system in some instances [2]. Preoperatively, almost all of the patients in our study were categorized into the SAA+ group or the SAA \pm group and were not treated with any medications known to have anticholinergic effects. Previous studies have focused on the relationship between SAA and exposure to anticholinergic drugs or their metabolites [17]; however, more recent findings indicate that the presence of endogenous AA can result from fever, acute infection [18] or stress [19] in ill elderly patients, independently from the use of such drugs. This supports our presumption that elevated SAA levels may be explained by the production of endogenous anticholinergic substances in response to stress. We also revealed that almost all of the postoperative serum samples could also be categorized into SAA \pm or
SAA-, which conflicted with our prediction that SAA levels would rise due to surgical stress. If our theory holds true, this suggests that the cognitive function was well preserved in some patients, and that in those cases, the normal function of the cholinergic system may have compensated for the increased AA [2]. There were no significant differences between the delirious and nondelirious groups concerning the preoperative SAA level, but SAA+ patients who remained SAA+ or became SAA \pm after surgery were more likely to develop postoperative delirium.

We conclude that delirious patients failed to compensate for the increase in AA, making it important to pay a close attention to the perioperative transition of the SAA level in relation to delirium, rather than focusing on a single SAA level. We excluded patients with characteristics thought to be related to delirium, so further investigation may be needed.

\section{LBD and AA}

LBD is a neurocognitive disorder that results in significant burdens for patients and their caregivers. It is associated with several symptoms, including visual hallucinations, fluctuating cognitive function and parkinsonism (i.e. core symptoms). Moreover, fragility to antipsychotic agents and rapid eye movement behavioral symptoms are symptoms highly suggestive of LBD [20]. In LBD, ACh downregulation is generally thought to be severer than that in AD. For example, Perry et al. [21] reported that downregulation of choline acetyltransferase activity was dominant in the temporal and parietal cortexes of patients with LBD, especially those with hallucinations. Perry and Perry [22] explained that the downregulation of $\mathrm{ACh}$ in the cortex introduced irrelevant intrinsic and sensory information, which caused the characteristic hallucinations of LBD. This was thought to be because ACh enhances cortical sensitivity to external stimuli and decreases corticocortical communication focused on attention (i.e. the neuronal signal-to-noise ratio). We speculate that the fragility to antipsychotic agents and the fluctuation of cognitive function (perhaps related with diurnal rhythm disturbance) in LBD are dependent on the anticholinergic burden [23].

Perry and Perry [22] also commented that the adverse events caused by ACh downregulation were similar to the actions of antimuscarinic drugs. Shiozaki et al. [24] also reported that muscarinic-1 receptors were relatively preserved in patients with LBD, while muscarinic-2 autoreceptors at presynaptic neurons were more severely dete- 
Fig. 2. The plasma steroid level is higher early in the morning and rapidly declines later. We hypothesized that this rapid decline might cause disinhibition of the immune system. Thus, the immune system may be activated in the afternoons, evenings and at night. If the levels of the steroids in the blood increase, any decline in these levels becomes more significant; this would lead to a more active inflammatory state and increased anticholinergic activity (AA, red arrow; colors in the online version). Courtesy of Hori et al. [2].

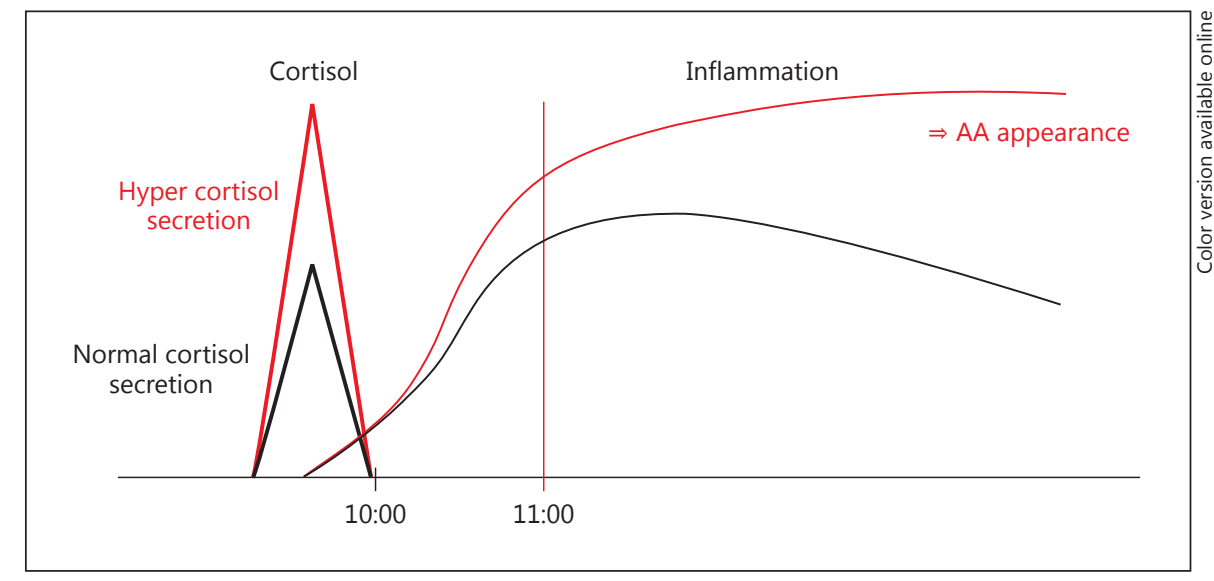

riorated than in $\mathrm{AD}$. They also reported that both receptors (neurons) were more deteriorated relative to those in normal controls. We speculated that relatively preserved muscarinic-1 postsynaptic receptors might compensate for the endogenous appearance of AA in LBD. So, even if cognitive dysfunction is not severe in LBD, ACh downregulation is relatively high because of the differences between $\mathrm{LBD}$ and $\mathrm{AD}$ in the distribution patterns of deteriorated $\mathrm{ACh}$ neurons. Therefore, AA should also be able to appear endogenously in LBD. Moreover, synergisms between amyloid and $\alpha$-synuclein are thought to cause additional, severe symptoms that accelerate the disease course [25]. The presence of both amyloid and $\alpha$-synuclein increases the inflammatory systems and levels of tau protein. Therefore, even if cognitive dysfunction in LBD is not severe, disease progression by downregulation of $\mathrm{ACh}$ is severer than in $\mathrm{AD}$. These considerations lead to the logical conclusion that the pathophysiology of both $\mathrm{AD}$ and $\mathrm{LBD}$ involves the development of $\mathrm{AA}$.

Another factor that is suggestive of the endogenous appearance of AA in LBD is that the autonomic parasympathetic nervous system is severely deteriorated in LBD [26]. This is attributable to the induction of AA in LBD, with the deteriorated autonomic parasympathetic nervous system causing increased activity of the hypothalamic-pituitary-adrenal axis and hypercortisolism [27, 28]. If inflammatory processes are caused by AA, it is plausible to conclude that corticosteroids should inhibit the action of AA, because of their anti-inflammatory properties. Despite this, corticosteroids have been reported to induce or increase AA in the brain [19]. Typically, the corticosteroid level in plasma is high early in the morning and rapidly declines thereafter. We theorized that this rapid decline might cause immune system disin- hibition, with immune activation in the afternoon, evening and night [2]. In fact, Wessa et al. [29] measured salivary cortisol levels at 7 intervals from morning to 8 p.m. in trauma-exposed subjects with and without posttraumatic stress disorder and in nonexposed controls and reported that at only awakening 15-60 min later was significantly lower in posttraumatic stress disorder patients than those in the other two groups. Bhagwagar et al. [30] reported that acutely depressed, medication-free subjects showed exaggerated release of cortisol in saliva in relation to awakenings. We speculate that in neurocognitive and neuropsychiatric disorders cortisol levels after awakenings, i.e. at morning level, might be affected. If the blood level of corticosteroids rises above normal, the subsequent decline in the corticosteroid levels can be expected to be larger, which is expected at the activated inflammatory state by the AA (fig. 2; courtesy of Hori et al. [2]). Therefore, it seems logical that even if AA does not appear early in the morning, it may appear by noon or later. This mechanism might explain why patients with delirium can appear calm in the morning and delirious in the late afternoon and at night, i.e. sundowning. We also believe that deteriorated parasympathetic autonomic nervous function causes AA in patients with LBD because of downregulation of $\mathrm{ACh}$, and that $\mathrm{AA}$ appears endogenously in LBD.

\section{Conclusion}

We have shown that AA could explain neurocognitive disorders other than $\mathrm{AD}$. In delirium, in which $\mathrm{ACh}$ downregulation does not occur at a critical level, AA appears by other AA inserts, including medicines, physical 
illnesses or mental stress. In LBD, we speculate that AA can appear regardless of whether cognitive dysfunction is severe. On the one hand, deteriorated patterns of ACh in $\mathrm{LBD}$ are different from those in $\mathrm{AD}$, and synergism between amyloid and $\alpha$-synuclein might create additional or severe symptoms that accelerate the disease course. On the other hand, disinhibition of reduced cortisol levels could result from severe autonomic parasympathetic dysfunction in LBD, thereby causing AA. However, further research is needed to confirm the basic science before progressing to the clinical usefulness. We need to demonstrate the basic science in a large cohort measuring SAA, cortisol and inflammatory activity, as well as stress measures. Anyway we believe that the appearance of AA is based on the downregulation of ACh.

\section{Acknowledgments}

Funding for this study was received from Eisai Co. Ltd., Daiichi Sankyo Inc. and Ono Pharmaceutical Co. Ltd.

\section{Disclosure Statement}

Koji Hori received lecture fees from Eisai Co. Ltd., Pfizer Japan Inc., Novartis Pharma KK, Daiichi Sankyo Inc., Ono Pharmaceutical Co. Ltd., Janssen Pharmaceutical KK, Yoshitomi Yakuhin Co. and Mitsubishi Tanabe Pharma Co. Mitsugu Hachisu received funding from Astellas Pharma Inc., Meiji Seika Pharma Co. Ltd., Dainippon Sumitomo Pharm Co. Ltd., Eli Lilly Japan KK and Shionogi \& Co. Ltd., and received lecture fees from Meiji Seika Pharma Co. Ltd. and Mitsubishi Tanabe Pharma Co.

\section{References}

1 Hori K, Konishi K, Akita R, Tani M, Tomioka H, Kitajima Y, Yokoyama S, Azuma K, Ikuse D, Akashi N, Yuda H, Hachisu M: Proposal of endogenous anticholinergic hypothesis in Alzheimer's disease (in Japanese). Jpn J Neuropsychopharmacol 2013;33:117-126.

2 Hori K, Konishi K, Tani M, Tomioka H, Akita R, Kitajima Y, Aoki M, Yokoyama S, Azuma K, Ikuse D, Akashi N, Hosoi M, Jinbo K, Hachisu M: Serum anticholinergic activity: a possible peripheral marker of the anticholinergic burden in the central nervous system in Alzheimer's disease. Dis Markers 2014;2014: 459013.

3 Hori K, Konishi K, Tani M, Tomioka H, Akita R, Kitajima Y, Aoki M, Kikuchi N, Ikuse D, Akashi N, Hosoi M, Jimbo K, Hachisu M: Why does the progression of Alzheimer's disease accelerate? Ann Psychiatry Ment Health 2014;2:1006.

-4 Whitehouse PJ, Price DL, Struble RG, Clark AW, Coyle JT, Delon MR: Alzheimer's disease and senile dementia: loss of neurons in the basal forebrain. Science 1982;215:1237-1239.

5 Hori K, Konishi K, Tomioka H, Tani M, Minegishi G, Tanaka H, Akita R, Yokoyama S, Oshio T, Hachisu M: Serum anticholinergic activity: a biomarker for rapid progression of Alzheimer's disease. J Autacoids 2012, DOI: org/10.4172/2161-0479.S4-001.

6 Tune L, Coyle JT: Serum levels of anti-cholinergic drugs in treatment of acute extrapyramidal side effects. Arch Gen Psychiatry 1980;37: 293-297.

7 Konishi K, Hori K, Tomioka H, Minegishi G, Tani M, Tanaka H, Akita R, Yokoyama S, Oshio T, Hachisu M: Donepezil abolishes anticholinergic activity in a patient with amnesia. Pharmacology 2013;91:86-91.
8 Folstein MF, Folstein SE, McHugh PR: 'Minimental state': a practical method for grading the cognitive state of patients for the clinician. J Psychiatr Res 1975;12:189-198.

-9 Morris JC, Cummings J: Mild cognitive impairment (MCI) represents early-stage Alzheimer's disease. J Alzheimers Dis 2005; 7: 235-239.

10 McKhann G, Drachman D, Folstein M, Katzman R, Price D, Stadlan EM: Clinical diagnosis of Alzheimer's disease: report of the NINCDS-ADRDA Work Group under the auspices of Department of Health and $\mathrm{Hu}$ man Services Task Force on Alzheimer's Disease. Neurology 1984;34:939-944.

11 Young J, Inouye SK: Delirium in older people. BMJ 2007;334:842-846.

12 McCusker J, Cole M, Dendukuri N, Han L, Belzile E: The course of delirium in older medical inpatients: a prospective study. J Gen Intern Med 2003;18:696-704.

13 Marcantonio ER, Rudolph JL, Culley D, Crosby G, Alsop D, Inouye SK: Serum biomarkers for delirium. J Gerontol A Biol Sci Med Sci 2006;61:1281-1286.

14 Lipowski ZJ: Delirium in the elderly patient. N Engl J Med 1989;320:578-582.

15 Plaschke K, Thomas C, Engelhardt R, Teschendorf P, Hestermann U, Weigand MA, Martin E, Kopitz J: Significant correlation between plas$\mathrm{ma}$ and CSF anticholinergic activity in presurgical patients. Neurosci Lett 2007;417:16-20.

-16 Kitajima Y, Yamaguchi K, Hori K, Murakami T, Someya A, Konishi K, Hara A, Hachisu M, Kajiyama Y, Nagaoka I, Inada E: The relationship between the perioperative transition of serum anticholinergic activity and postoperative delirium in patients undergoing esophagectomy and gastrectomy. Juntendo Med J 2014;60:147-150.
17 Tune L, Carr S, Hoag E, Cooper T: Anticholinergic effects of drugs commonly prescribed for the elderly: potential means for assessing risk of delirium. Am J Psychiatry 1992;149: 1393-1394.

18 Flacker JM, Lipsitz LA: Serum anticholinergic activity changes with acute illness in elderly medical patients. J Gerontol A Biol Sci Med Sci 1999;54:M12-M16.

19 Plaschke K, Kopitz J, Mattern J, Martin E, Teschendorf P: Increased cortisol levels and anticholinergic activity in cognitively unimpaired patients. J Neuropsychiatry Clin Neurosci 2010;22:433-441.

20 McKeith IG, Dickson DW, Lowe J, Emre M, O'Brien JT, Feldman H, Cummings J, Duda JE, Lippa C, Perry EK, Aarsland D, Arai H, Ballard CG, Boeve B, Burn DJ, Costa D, Del Ser T, Dubois B, Galasko D, Gauthier S, Goetz CG, Gomez-Tortosa E, Halliday G, Hansen LA, Hardy J, Iwatsubo T, Kalaria RN, Kaufer D, Kenny RA, Korczyn A, Kosaka K, Lee VM, Lees A, Litvan I, Londos E, Lopez OL, Minoshima S, Mizuno Y, Molina JA, Mukaetova-Ladinska EB, Pasquier F, Perry RH, Schulz JB, Trojanowski JQ, Yamada M; Consortium on DLB: Diagnosis and management of dementia with Lewy bodies: third report of the DLB Consortium. Neurology 2005; 65:18631872.

21 Perry EK, Marshall E, Kerwin J, Smith CJ, Jabeen S, Cheng AV, Perry RH: Evidence of a monoaminergic-cholinergic imbalance related to visual hallucinations in Lewy body dementia. J Neurochem 1990;55:1454-1456.

22 Perry EK, Perry RH: Acetylcholine and hallucinations: disease-related compared to drug-induced alterations in human consciousness. Brain Cogn 1995;28:240-258. 
23 Konishi K, Hori K, Tomioka H, Tani M, Tanaka H, Akita R, Yokoyama S, Oshio T, Hachisu M: Is anticholinergic activity related with Parkinson's disease? Psychogeriatrics 2012;12:214.

24 Shiozaki K, Iseki E, Uchiyama H, Watanabe Y, Haga T, Kameyama K, Ikeda T, Yamamoto T, Kosaka K: Alterations of muscarinic acetylcholine receptor subtypes in diffuse Lewy body disease: relation to Alzheimer's disease. J Neurol Neurosurg Psychiatry 1999;67:209-213.

25 Jaya JC, Chatterjee P, Sengupta N: Cross dimerization of amyloid- $\beta$ and $\alpha$-synuclein proteins in aqueous environment: a molecular dynamics simulations study. PLoS One 2014; 9:e106883.
6 Stubendorff K, Aarsland D, Minthon L, Londos E: The impact of autonomic dysfunction on survival in patients with dementia with Lewy bodies and Parkinson's disease with dementia. PLoS One 2012;7:e45451.

27 Herrscher RF, Kasper C, Sullivan TJ: Endogenous cortisol regulates immunoglobulin Edependent late phase reactions. J Clin Invest 1992;90:596-603.

28 Masson GS, Nair AR, Dange RB, Silva-Soares PP, Micheline LC, Francis J: Toll-like receptor 4 promotes autonomic dysfunction, inflammation and microglia activation in hypothalamic paraventricular nucleus: role of endoplasmic reticulum stress. PLoS One 2015;10: e0122850.
29 Wessa M, Rohleder N, Kirschbaum C, Flor H: Altered cortisol awakening response in posttraumatic stress disorder. Psychoneuroendocrinology 2006;31:209-215.

-30 Bhagwagar Z, Hafizi S, Cowen PJ: Increased salivary cortisol after waking in depression. Psychopharmacology (Berl) 2005;182:54-57. 